\title{
INTRODUCTION
}

\section{Dispositifs numériques et organisations. Entre permanences, tensions et changements}

NATHALie Pinede

Les mutations des organisations se poursuivent aujourd'hui, dans un mouvement de «mise en numérique " généralisé et d'innovations technologiques, concernant tout autant les modalités de production que les transactions marchandes, les processus communicationnels ou encore la géométrie des collaborations. La vision d'une «entreprise du futur» (Besson, 2015) illustre bien les dynamiques de changements à l'œuvre, ainsi que les projections qui les accompagnent. Cependant, au-delà de multiples effets d'annonces, il est important de rappeler que le déploiement des dispositifs numériques s'inscrit dans les trajectoires organisationnelles, soit dans un «long processus de négociations et d'ajustements» (Jemine, 2017, p. 58) entre stratégies, acteurs, usages et techniques, processus non exempt d'imaginaires et d'idéologies, sous-tendu par l'éternel mythe du progrès ainsi que par les promesses du numérique, telles qu'elles peuvent être portées notamment par l'intelligence artificielle ou encore l'ubiquité connectée.

Au-delà de formes d'invisibilité et de banalisation (Andonova, 2015), l'omniprésence des dispositifs numériques dans nombre d'organisations est donc une réalité, pour autant extrêmement diversifiée. Ceux-ci peuvent être 
dédiés à la gestion ou à l'analyse de données (intranets, ERP1, datamining...), par exemple en vue de la prise de décision, aux échanges info-communicationnels (réseaux sociaux, messagerie électronique...) ou encore modifier profondément la nature même des activités (dématérialisation de services, colloboration humain/robot - cobotique-, recours à la réalité virtuelle, etc.). Avec cette diversification, les limites de l'organisation deviennent également poreuses, le rapport « interne »/ « externe » (à l'organisation) se fait plus ambigu et d'une façon générale, la place de l'humain, plus diffuse et instable, entre inscription et flux, entre présence physique dans la structure et autonomie à distance, voire délocalisation de certaines tâches fragmentées et ingrates (ce qu'illustre le concept de micro-travail - Casilli et al., 2019).

Dans ces environnements de travail où le numérique tend à reconfigurer les modalités d'action, de collaboration, d'échanges, de production, la place de l'humain (en tant que "travailleur», usager, client, etc.) peut s'analyser à différentes échelles et sur différents plans d'intervention. A l'échelle individuelle, on peut s'intéresser aux rapports concrets aux technologies, pouvant se traduire par une réorganisation du poste de travail, de nouvelles interactions ou collaborations techno-instrumentées, etc. A l'échelle organisationnelle, il s'agit d'envisager la place de l'humain en tant que membre d'un collectif à repenser autour de et avec la présence croissante de dispositifs numériques diversifiés. Enfin, à l'échelle sociétale, des macro-éléments de contexte (cadre institutionnel, contraintes et opportunités réglementaires, environnementales...), impliquant l'humain dans ses attentes, désirs et craintes face au numérique, sont susceptibles de modifier le cadre d'action de l'organisation, et vice-versa. Bien entendu, ces différents niveaux sont en interrelations et s'imbriquent de façon systémique.

Dans le cadre de cet appel, nous focaliserons sur l'un de ces niveaux, en l'occurrence les enjeux collectifs soulevés par la présence de dispositifs numériques pluriels dans les organisations, entre permanences et transitions, opportunités et risques. Plusieurs questionnements peuvent être envisagés à cette échelle. Ainsi la question du travail émerge-t-elle comme une question cruciale : comment celui-ci se redéfinit-il dans les environnements organisationnels en prise avec le numérique (Bidet et al., 2017) ? Comment, à cette aune, se redessinent métiers et compétences, par exemple entre compétences techniques et communicationnelles (Bouillon, 2015) ? Comment se restructurent les collectifs de travail (Zacklad, 2015), dans et hors « les murs » de l'organisation, entre participatif supporté par des dispositifs numériques et responsabilité individuelle accrue?

\footnotetext{
${ }^{1}$ Enterprise Resource Planing
} 
Une autre question concerne la prise de décision et le management: comment les nouveaux modes de management par les données et les traces (Rouvroy et Berns, 2013; Vayre, 2018) peuvent-ils venir bousculer les pratiques? Avec quelle prise en charge (ou pas) des interrogations éthiques posées par les dispositifs numériques, par exemple en ce qui concerne les données (Balicco et al., 2018) mais aussi des risques sociaux afférents, en matière d'inclusion / exclusion ou de vulnérabilités numériques (Laborde et al., 2019)?

On peut se demander également si les stratégies numériques se développant dans les organisations s'accompagnent de démarches en matière de d'accompagnement au changement, en lien avec les transformations et transitions technologiques. Voit-on dès lors émerger des formes d'alignement (ou de dés-alignement) entre stratégies, dispositifs numériques et usages (Pinède, 2017)?

Ces questionnements ne sont évidemment pas exhaustifs mais tracent quelques lignes de force sur les permanences, tensions, changements à l'œuvre en lien avec la place croissante des dispositifs numériques dans les organisations. Les six textes qui composent au final ce dossier de Les Cabiers $d u$ Numérique en restituent un panorama tout à la fois homogène et contrasté. Trois axes ont été particulièrement privilégiés : ce que ces dispositifs numériques font aux métiers, reconfigurant les pratiques et la place de ceux-ci au sein des organisations ou des processus de production ; les risques associés, en matière de précarisation ou au plan techno-social ; enfin, les phénomènes d'hybridation numérique à l'œuvre et la relativisation du pouvoir attribué aux dispositifs numériques.

Les deux premiers textes focalisent donc plus particulièrement sur les mutations des métiers et des identités professionnelles en lien avec le numérique. Dans son article intitulé «L'identité professionnelle des localiers à l'heure des mutations économiques et numériques de la presse locale. Vers un journalisme de services ? ", Pauline Amiel montre, à partir d'un travail de terrain appuyé sur des entretiens avec des journalistes de cinq titres de presse locale, sur une observation dans une rédaction ainsi que sur l'analyse d'un corpus de documents issus de groupes de presse, comment la double évolution (économique et numérique) des entreprises de presse vient remodeler l'identité professionnelle des localiers. Par localier, P. Amiel entend le journaliste travaillant pour un titre de presse locale. Parmi les nombreux enseignements issus de son travail de terrain, re-situé dans une perspective historique, l'on voit notamment comment les deux caractéristiques principales de l'identité professionnelle des localiers, à savoir la relation de proximité avec son lectorat et l'ancrage fort dans un territoire, sont bousculées par la mise en numérique 
des organismes de presse ainsi que par leur réorientation économique. L'évolution des entreprises de presse locale en groupes multimédias insuffle une diversification des activités et un repositionnement au sein du groupe, la mise en numérique influant quant à elle l'organisation du travail, la transversalité des activités et les modes de rationalité associés. Ainsi, les localiers peuvent-ils se retrouver collecteurs de données au service du groupe. Dans ce cadre, le lien établi avec un lectorat de proximité est remis en cause par des logiques d'audience, alors que la "polyphonie énonciative «ne cesse de croître. Journalisme de service et journalisme de solutions deviennent alors de nouvelles formes de (re)légitimation de l'identité professionnelle des localiers, non sans distorsions et paradoxes.

Le deuxième texte, proposé par Rémi Adjiman, se penche quant à lui sur « le cas du monteur son », "au cœur de l'évolution de la chaine de fabrication cinématographique ». A contrario du texte précédent, où un métier «traditionnel» (celui de journaliste et plus précisément, de localier), se trouve mis en tensions par les évolutions économiques et numériques, l'émergence du métier de «monteur son » à partir des années 1980 est pour sa part fortement liée aux innovations technologiques (diffusion multicanal) et à la mise en numérique de la création cinématographique. Les tensions et résistances concernent dès lors l'irruption d'un nouvel acteur dans une chaîne de fabrication aux rôles précédemment bien définis, reconnus et ordonnés, et ce, depuis quasiment un siècle. Le monteur son s'est donc posé en élément perturbateur, appelant de nouvelles fonction, le recours à de nouveaux outils et la modification des méthodes de travail. En s'appuyant notamment sur une série d'entretiens avec des professionnels du secteur, l'auteur relève plusieurs points saillants en lien avec cette évolution technologique et numérique autour du son. Ainsi, si l'environnement technologique ouvre de nouveaux espaces de créativité, un processus de rationalisation, de division et de standardisation des tâches, dans une logique de type workflow, s'est peu à peu imposé, amenant dès lors de nouvelles contraintes. De même la possibilité d'un déport de la prise de décision en bout de chaîne modifie les méthodes de travail, en obligeant à un découpage précis des éléments sonores. La collaboration et la communication entre le réalisateur et le monteur son peuvent s'avérer également délicates. Enfin, la logique économique prévalant actuellement dans la production cinématographique et télévisuelle, en lien avec le processus de rationalisation vie le numérique et l'injonction à l'efficacité, met en tension la création artistique, le monteur son devant plutôt privilégier l'occupation affichée d'un espace sonore à la recherche, plus longue et discrète, d'effets d'ambiance feutrés. 
Il est aussi question de nouveaux métiers dans le texte proposé par Benjamin Lorre, ou plus exactement, il est question de nouvelles formes d'organisation du travail et de collaboration à travers les espaces de co-travail ou co-working. Le travailleur indépendant est au centre de ce milieu d'activités partagé et hyper-technicisé (Zacklad, 2018) qu'est l'espace de co-travail. Celui-ci affiche des valeurs de partage, de coopération, de convivialité, propices au développement d'une intelligence collective et à une culture du projet; il se présente également comme un moyen de rompre l'isolement des travailleurs. Néanmoins, au-delà de cette vision positive, la réalité s'avère plus contrastée. C'est ce que l'auteur met en évidence à partir d'un travail de terrain (observation empirique et entretiens semi-directifs) réalisé dans un espace de co-travail parisien. Autour des enjeux sociotechniques, socioéconomiques et organisationnels liés à ces espaces de travail, se développent des formes d'ambivalence, entre créativité et dépendance numérique, dynamiques collectives et autonomie des pratiques, dans une approche utilitariste de consommation de ressources. Ainsi, dans les espaces de co-travail, se créent les conditions d'émergence d'un "précariat», par analogie et distanciation avec le «salariat» et la «rassurance» qui peut l'accompagner. Le travailleur indépendant, s'il peut trouver dans ces lieux équipés des points d'ancrage collectifs, reste malgré tout soumis à des impératifs de flexibilité et à une fragilité de son statut qui représentent des vulnérabilité constantes.

Ces vulnérabilités peuvent prendre la forme de véritables risques en contextes organisationnels. C'est ce que présentent Nicolas Oliveri et Nicolas Pélissier dans leur texte intitulé "Repenser les dispositifs numériques des organisations au prisme des risques technosociaux (RTS)». Pouvant se manifester sous forme de syndrome d'épuisement technologique (SET), les risques technosociaux (RTS), déclinaisons des risques psychosociaux (RPS) en lien avec les environnements de travail numériquement équipés, représentent aujourd'hui une réalité. Si celle-ci est bien perçue et dénoncée par un certain nombre de chercheurs, notamment en sciences de l'information et de la communication (Carayol et al., 2016 ; Boudhokane-Lina, Felio, 2015), elle peine encore à être pleinement reconnue dans les entreprises, au nom d'imaginaires technophiles toujours prégnants. Plaidant pour une véritable "pédagogie numérique en direction des managers et des collaborateurs ", les auteurs proposent donc une série d'outils et de démarches de diagnostic qui ont pour vocation d'accompagner la prise en charge des RTS dans les entreprises. Ainsi, l'effet Shelley et son indice associé permettent de dresser un premier constat des effets indésirables et non prévus des TIC dans les entreprises. Un autre outil, appelé "Cycle d'apparition des RTS», donne en six étapes les moyens d'identifier les conditions d'émergence des RTS, pouvant aller jusqu'à une manifestation sous forme de SET. Si le diagnostic permet de dresser un 
nécessaire constat, agir de façon préventive constitue également un volet important pour anticiper les RTS, accompagner les entreprises dans leur développement technologique et numérique et favoriser le bien-être au travail des personnes en activité, immergées dans des environnements technonumériques.

Remettre l'humain au centre des dispositifs techniques, c'est aussi l'objectif du texte d'Antoine Henry sur l' "Hybridation numérique d'une organisation énergétique ». L'auteur mobilise notamment le concept de «faisabilité sociotechnique", soit le "processus d'insertion et de diffusion sociale qui s'inscrit dans une échelle de temps [et] qui nécessite un certain nombre de phases» (Zelem, 2012, p. 4) pour mettre en évidence les phénomènes d'hybridation à l'œuvre, produits d'ajustements entre numérique et logiques organisationnelles et individuelles, et à ce titre, inscrits dans une dynamique temporelle tout à la fois structurante et labile. A partir d'une étude de cas menée sous forme d'observation participante et d'entretiens dans une grande organisation du secteur énergétique français, l'auteur montre les multiples effets de déplacement (par exemple, nouveau poids politique de la Direction des systèmes d'information au détriment d'autres directions "historiques»), de substitution (augmentation de la place des médiations numériques dans l'entreprise) ou encore de réagencements (nouvelles formes de rationalisation « tayloriste » de l'activité mais aussi nouvelles formes d'action collective). Ainsi, A. Henry brosse-t-il un tableau où le temps révèle les transformations à l'œuvre quand la technique rencontre l'individu (et vice-versa...).

Enfin, le texte qui clôt ce dossier illustre les modalités de résistance de l'humain à l'injonction du pilotage par la technique et les données. Dans son article intitulé «L'outil face au manager: le contrôle du travail à l'ère du numérique, un terrain controversé ? ", Gregory Jemine met la focale sur le rôle du manager de proximité dans la relativisation de l'autorité du numérique dans les organisations. Cette position s'inscrit à rebours de la majorité des écrits issus de la littérature scientifique qui présentent généralement l'usage des technologies numériques pour le management (en l'occurrence ici, des données et des possibilités de métrique qui en découlent) selon ce que l'auteur appelle quatre "pseudo-paradigmes » (de façon simplifiée, en tant qu'indispensable appui, source d'affaiblissement, voire de substitution, ou encore moyen panoptique de surveillance). Or, ces écrits, pour la plupart, font peu de place à la parole des managers de proximité et à une analyse pragmatique de leurs situations d'activité en lien avec les techniques. Le travail empirique et qualitatif réalisé par G. Jemine dans une compagnie d'assurances belge apporte justement un éclairage, situé et ancré, sur le rôle du manager de proximité. Cette étude fait ainsi apparaître les négociations et perceptions relativisées de certains 
indicateurs de « performance » qui, en l'occurrence, ne seront pas pris « au pied de la lettre par les managers. Un autre exemple tout à fait significatif concerne les contradictions entre l'incitation au monitoring via des outils de pilotage et de contrôle, d'une part et, d'autre part, l'incitation à adhérer à un projet privilégiant une culture du management par la confiance et l'autonomie, projet auquel souscrivent plutôt les managers de proximité... L'intérêt de cette étude empirique consiste donc à remettre au premier plan les capacités d'agir et de réagir des acteurs, y compris dans des écosystèmes numériques puissants.

L'ensemble de ces textes montre donc la complexité de la problématique des dispositifs numériques dans les organisations, entre transformations, risques, mais aussi hybridation et relativisation d'une certaine propension au déterminisme technique. L'humain doit et peut rester au cœur de ces dispositifs numériques...

Remerciements : la coordinatrice du numéro remercie vivement le comité de lecture de ce numéro pour le travail de lecture et de sélection des textes.

\section{Références citées}

Andonova, Y. (2015). De l'invisibilité des dispositifs numériques à la légitimation de la communication en entreprise. Sociologies pratiques, vol. 1, n³0, p. 43-52.

Balicco, L., Broudoux, E., Chartron, G., Clavier, V. et Pailliart, I. (2018). L'éthique en contexte info-communicationnel numérique. Déontologie, régulation, algorithme, espace public, Louvain-La-Neuve, De Boeck Supérieur.

Besson, M. (dir.) (2015). Entreprise du futur. Les enjeux de la transformation numérique. Livre Blanc, Paris, Institut Mines-Télécom. Disponible sur: https://www.marsouin.org/IMG/pdf/imt livreblanc.pdf

Bidet, A., Datchary, C., Gaglio, G. (2017). Quand travailler, c'est s'organiser. La multi-activité à l'ère numérique, Paris, Presses des Mines.

Boudokhane-Lima, F., Félio, C. (2015). Les usages professionnels des TIC: des régulations à construire. Communication et organisation, $\mathrm{n}^{\circ} 48$.

Bouillon, J-L. (2015). «Technologies numériques d’information et de communication et rationalisations organisationnelles: les «compétences numériques » face à la modélisation ». Les Enjeux de l'information et de la communication, vol. 16, n¹, p. 89-103.

Carayol, V. et al. (2016). La laisse électronique, les cadres débordés par les TIC. Pessac, Maison des Sciences de l'Homme d'Aquitaine.

Casilli, A. A., Tubaro, P., Le Ludec, C., Coville, M., Besenval, M., Mouhtare, T., Wahal, E. (2019). Le Micro-travail en France. Derrière l'automatisation de nouvelles précarités au 
16 Les cahiers du numérique $-\mathrm{n}^{\circ}$ 4/2019

travail ? Rapport Final Projet DiPLab «Digital Platform Labor». Disponible sur http://diplab.eu.

Jemine, G. (2017). Déploiement de dispositifs numériques au sein de nouvelles formes d'organisation: de l'émergence à la stabilisation. Sociologies pratiques, vol. $1, \mathrm{n}^{\circ} 34$, p. 49-59.

Laborde, A. (coord.) (2019). Le numérique : nouvelles sources d'incivilités au travail. Expériences, usages, droits, témoignages, définitions. Livre blanc. Disponible sur https://www.ubordeaux-montaigne.fr/fr/actualites/recherche/le-numerique-nouvelles-sources-dincivilites-au-travail.html

Pinède, N. (2017). "Analyse stratégique des sites web. Approche par l'alignement », Dans S. Rouquette (dir.), Site internet : audit et stratégie, Louvain-la Neuve, De Boeck, p. 51-76.

Rouvroy, A., Berns, T. (2013). Gouvernementalité algorithmique et perspectives d'émancipation. Le disparate comme condition d'individuation par la relation ?. Réseaux, n¹77, p. 163-196.

Vayre, J. (2018). Comment décrire les technologies d'apprentissage artificiel : le cas des machines à prédire. Réseaux, 211(5), p. 69-104.

Zacklad, M. (2015). Régimes de coopération dans les comportements collectifs médiatisés. Dans S. Alemanno (dir). Communication organisationnelle, management et numérique, Paris, L'Harmattan, p. 153-158.

Zacklad M. (2018). Vers une permaculture des milieux d'activités partagés. Actes du Colloque de Cerisy "Quelles communications, quelles organisations à l'ère du numérique 》.

Zelem, M. (2012). Les énergies renouvelables en transition : de leur acceptabilité sociale à leur faisabilité sociotechnique. Revue de l'Énergie, nº 610, p. 1-8. 